\title{
UCRL-CONF-227801
}

LAW RENCE LIVERMORE N A TIO N A L LABORATORY

\section{Adaptive Wavefront Calibration and Control for the Gemini Planet Imager}

L. A. Poyneer, J.-P. Veran

February 6, 2007

Adaptive Optics: Analysis and Methods

Vancouver, Canada June 18, 2007 through June 20, 2007 
This document was prepared as an account of work sponsored by an agency of the United States Government. Neither the United States Government nor the University of California nor any of their employees, makes any warranty, express or implied, or assumes any legal liability or responsibility for the accuracy, completeness, or usefulness of any information, apparatus, product, or process disclosed, or represents that its use would not infringe privately owned rights. Reference herein to any specific commercial product, process, or service by trade name, trademark, manufacturer, or otherwise, does not necessarily constitute or imply its endorsement, recommendation, or favoring by the United States Government or the University of California. The views and opinions of authors expressed herein do not necessarily state or reflect those of the United States Government or the University of California, and shall not be used for advertising or product endorsement purposes. 


\title{
Adaptive Wavefront Calibration and Control for the Gemini Planet Imager
}

\author{
Lisa A. Poyneer \\ Lawrence Livermore National Laboratory, 7000 East Avenue, Livermore, California 94550 \\ poyneer1@llnl.gov \\ Jean-Pierre Véran \\ Herzberg Institute of Astrophysics, 5071 West Saanich Road, Victoria, British Columbia, Canada V9E2E7 \\ Jean-Pierre.Veran@nrc-cnrc.gc.ca
}

\begin{abstract}
Quasi-static errors in the science leg and internal AO flexure will be corrected. Wavefront control will adapt to current atmospheric conditions through Fourier modal gain optimization, or the prediction of atmospheric layers with Kalman filtering.
\end{abstract}

(C) 2007 Optical Society of America

OCIS codes: (010.1080) Adaptive Optics, (350.1260) Astronomical optics

\section{Introduction}

The Adaptive Optics (AO) system of the Gemini Planet Imager (GPI) must achieve ambitious performance goals to enable the direct imaging detection of extrasolar planets. As part of this, the AO system will run at up to $2 \mathrm{kHz}$ with 44 subapertures across the Gemini pupil. Though substantial performance gains can come from high frame rates and small subapertures, GPI's AO system will use adaptive wavefront control methods to obtain the best performance under changing atmospheric conditions. Furthermore, the sensitivity of the science image to small quasi-static errors requires adaptive calibration of the $\mathrm{AO}$ wavefront controller. These two areas have been a focus of the preliminary design phase of the GPI instrument. The core components of the AO system which are involved are shown in Fig. 1.

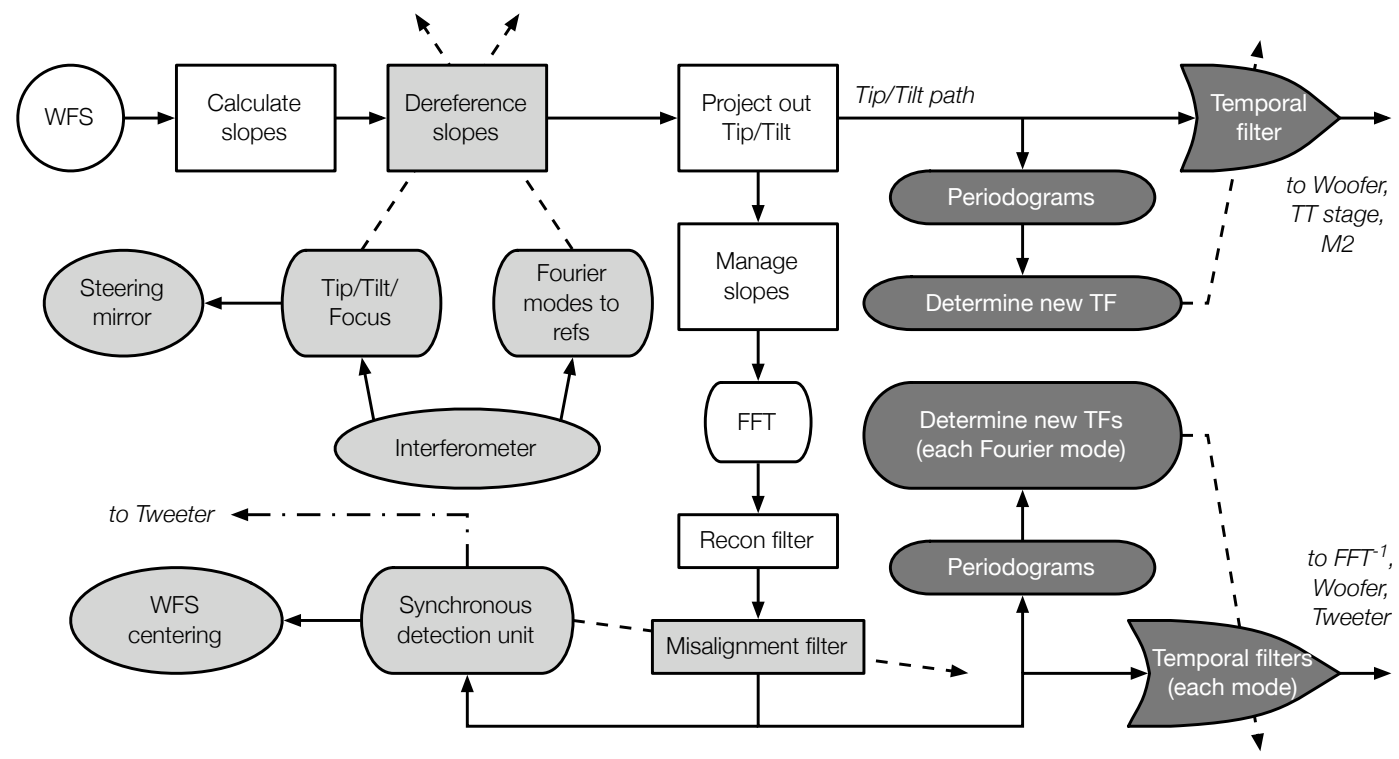

Fig. 1. Conceptual block diagram of the relevant portion of the GPI AO control system. Light grey elements on the left side execute adaptive calibration of the AO system. Dark grey elements on the right implement adaptive wavefront control based on atmospheric statistics. 


\section{Adaptive temporal filtering of Fourier modes}

At each time step, the wavefront is reconstructed with the Fourier Transform Reconstruction (FTR) [1]. In the figure, this is the center column of white elements. In addition to substantial computational efficiency, Fourier modes are desirable for controller optimization. As clearly expressed in the PSF expansion [2], at high Strehl ratios, the spatial frequencies (i.e. Fourier modes) of the phase aberration directly correspond to scattered light at spatial locations in the PSF. This means that optimization of each Fourier mode leads to optimization of each spatial location in the science image. Under the assumption of wide-sense-stationarity of the random processes of the atmosphere and WFS noise, the Fourier modes are uncorrelated with each other. This can be exploited to allow independent temporal filtering of each Fourier mode without consideration of modal correlations.

System telemetry is analyzed to determine the best temporal filters. After reconstruction, the residual phase (in the form of complex-valued Fourier modal coefficients) is buffered. The temporal PSD for each Fourier mode is estimated with the periodogram method from the last few seconds of data. The temporal characteristics of the atmosphere are then used to determine the best temporal filter for each mode. These temporal filters are implemented in the Fourier domain and produce the best estimate of the full phase which must be compensated. This full phase is then compensated in a Woofer-Tweeter arrangement with a MEMS mirror and a low-order DM [3].

GPI's baseline adaptive filter is determined by modal gain optimization (developed by Gendron and Léna [4]) of a leaky integrator. When used with FTR, this method is termed Optimal Fourier Control (OFC) [5]. OFC rapidly (at up to $\sim 2 \mathrm{~Hz}$ ) determines the optimal gain for each mode based on the atmospheric signal and WFS noise power levels. The varying levels of power in the atmosphere, when coupled with discrete layers moving in different directions, require a large range of optimal gain as a function of spatial frequency [6]. In addition, OFC can compensate for any uncalibrated gains due to MEMS influence functions.

We have recently developed a predictive temporal filtering scheme, termed Predictive Fourier Control (PFC) [7]. This method generates predictive filters under the assumption of frozen flow atmospheric turbulence. PFC builds upon the Kalman filtering formalism for closed-loop AO control that was developed by Le Roux, et al [8]. Under frozen flow, each layer of turbulence produces a narrow spike in the temporal PSD of a given Fourier mode. This concentration of power in a narrow region allows accurate detection of layers from closed loop telemetry. When coupled with the Kalman framework, the optimal predictive filter has a compact form which effectively notches out each atmosphericlayer peak. The exact coefficients for each predictive filter are determined by an iterative solution of the algebraic Riccati equation. Compared to the performance of a generic (unoptimized, unadaptive) controller, OFC reduces the scattered light everywhere in the PSF. When a dominant wind layer is present, the residual error forms a 'butterfly' or 'hourglass' shape in the PSF. The prediction of PFC removes this characteristic shape, leaving a PSF which is radially symmetric and has no evidence of wind direction. In addition, PFC has lower noise propagation than OFC. While OFC fits easily into GPI's baseline off-the-shelf (OTS) control computer, PFC would require between 1 to 2 GFLOPs/sec from the computer, depending on the number of layers. This amount may push the limits of current OTS technology.

Tip and tilt will always be removed from the main FTR/adaptive filtering path. They will be controlled separately adaptive temporal filtering. In particular, this should allow for the reduction of vibration, as has been recently experimentally demonstrated by Petit, et al [9].

\section{Adaptive calibration}

Dynamic errors which the AO system corrects will not be the limiting factor in final GPI science image contrast. Instead, chromatic errors and the slowly-varying phase errors which the AO system either causes (due to uncalibrated instrument flexure) or cannot fix (errors in the science leg) will limit performance. This is the motivation for the builtin calibration system of an interferometer in the science leg of the instrument [10]. The interferometer will provide measurements of the slowly varying phase aberrations present in the science beam after the final pupil stop in the apodized Lyot coronagraph. The calibration unit will provide the AO system with tip, tilt and focus measurements, and a spatial map of residual phase errors on the same sampling as the MEMS actuator grid. Interferometer data will be used in two ways to update the calibration of the AO system.

First, reference slope values will be manipulated. For low-order modes the values of the reference slopes which are necessary are simple: tip and tilt correspond to a constant offset in the slopes, and focus to a linear term. For the higher-order spatial mapping of the residual phase error, a Fourier algorithm is used. This algorithm is in essence the inverse of FTR. For each of the $\mathrm{x}$ - and $\mathrm{y}$-slopes, a filter is applied which captures the measurement process of the Shack Hartmann (see [5] for a specification of such a filter). Any additional filtering, such as fine $\mathrm{x}$ - and y-alignment, or compensation for a known linear process in the interferometer's measurement, can be done at the same time. The 
new values are added to the present reference slopes in a sensible fashion. We have demonstrated the use of this algorithm at the UCSC LAO Extreme AO testbed [11]. We have verified that this calibration method can be used to manipulate the science residual phase at the sub-nm RMS level, and to correctly fix errors.

The second method of calibration is actually an adjustment of system alignment using steering mirrors. In particular, tip, tilt and focus can be compensated for given optics which exist in the AO system. This type of correction is especially important for tip and tilt. Because the WFS uses $2 \times 2$ pixel quadcells, there is a limit on the linear range of the WFS. If the reference slopes are too large, the WFS will operate off-null and push actual slope measurements of the dynamic atmosphere out of the linear range of the WFS.

Flexure can manifest as a dynamic error, wherein the quality of AO correction is reduced. An example is a drift of the MEMS actuators relative to the WFS lenslet array. This will produce not a quasi-static error but a small increase in dynamic error in the system. It will be possible in GPI that flexure-induced misalignments will lead to reduced AO performance, making the dynamic error terms significant. Detection and correction of dynamic errors requires a method separate from the calibration interferometer. Instead, the AO system itself will be used. The alignment of the pupil to the WFS will be tracked with the WFS intensity measurements. Alignment of the lenlets to the MEMS will be done by injecting known patterns (with both spatial and temporal characteristics) onto the MEMS and analyzing the resulting AO telemetry. Such an approach, in a static manner, is used to align the AO system. For GPI we have developed a robust optimization algorithm [11] which uses two different Fourier modes placed on the mirror to estimate the registration between the MEMS actuators and the lenslet array.

The method of synchronous detection [12] is a prime candidate for enabling such calibration during closed-loop. In this method, the desired signal on the MEMS is modulated temporally, which enables closed-loop on-sky operation. The resulting AO telemetry is demodulated and then processed as required. For GPI it remains to be determined whether or not it is necessary to monitor lenslet-MEMS registration during science imaging. Instead, we have adopted a baseline strategy of injecting signals during the regular intervals where the science instrument is shuttered, but the AO system remains in closed loop. Lenslet-MEMS registration will be monitored and then corrected by adjusting physical items (e.g. steering the pupil with mirrors) or by using a misalignment compensation filter [13].

\section{Acknowledgments}

This work was performed under the auspices of the U.S. Department of Energy by the University of California, Lawrence Livermore National Laboratory under contract No. W-7405-Eng-48. The document number is UCRLCONF-227801. This work has been supported by the National Science Foundation Science and Technology Center for Adaptive Optics, managed by the University of California at Santa Cruz under cooperative agreement No. AST 9876783.

\section{References}

1. L. A. Poyneer, D. Gavel, and J. Brase, "Fast wave-front reconstruction in large adaptive optics systems with use of the Fourier transform," J. Opt. Soc. Am. A 19, 2100-2111 (2002).

2. M. D. Perrin, A. Sivaramakrishnan, R. Makidon, B. Oppenheimer, and J. Graham, "The structure of high Strehl ratio point-spread functions," Ap. J. 596, 702-712 (2003).

3. J.-F. Lavinge et al, "Woofer - Tweeter control algorithm for the Gemini Planet Imager," submitted to this conference.

4. E. Gendron and P. Léna, "Astronomical adaptive optics I. Modal control optimization,” Astron. Astrophys. 291, 337-347 (1994).

5. L. A. Poyneer and J.-P. Véran, "Optimal modal Fourier transform wave-front control," J. Opt. Soc. Am. A 22, 1515-1526 (2005).

6. L. A. Poyneer and B. Macintosh, "Optimal Fourier Control performance and speckle behavior in high-contrast imaging with Adaptive Optics," Opt. Exp. 14, 7499-7514 (2006).

7. L. A. Poyneer, B. Macintosh, and J.-P. Véran, "Fourier transform wavefront control with adaptive prediction of the atmosphere," J. Opt. Soc. Am. A (submitted 2006).

8. B. Le Roux, J.-M. Conan, C. Kulcsar, H.-F. Raynaud, L. Mugnier, and T. Fusco, "Optimal control law for classical and multiconjugate adaptive optics," J. Opt. Soc. Am. A 21, 1261-1276 (2004).

9. C. Petit, J.-M. Conan, C. Kulcsar, H.-F. Raynaud, T. Fusco, J. Montri, and D. Rabaud, "First laboratory demonstration of closed-loop Kalman based optimal control for vibration filtering and simplified MCAO," in Advances in Adaptive Optics II, B. L. Ellerbroek and D. B. Calia, eds., Proc. SPIE 6272, p. $62721 \mathrm{~T}$ (2006).

10. J. K. Wallace, R. Bartos, S. Rao, R. Samuele, and E. Schmidtlin, "A laboratory experiment for demonstrating post-coronagraph wavefront sensing and control for extreme adaptive optics," in Advances in Adaptive Optics II, B. L. Ellerbroek and D. B. Calia, eds., Proc. SPIE 6272, p. 62722L (2006).

11. L. A. Poyneer, J.-P. Véran, D. Dillon, S. Severson, and B. Macintosh, "Wavefront control for the Gemini Planet Imager," in Advances in Adaptive Optics II, Proc. SPIE 6272, p. 62721N (2006).

12. S. Esposito, R. Tubbs, A. Puglisi, S. Oberti, A. Tozzi, M. Xompero, and D. Zanotti, "High SNR measurement of interaction matrix on-sky and in lab," in Advances in Adaptive Optics II, B. L. Ellerbroek and D. B. Calia, eds., Proc. SPIE 6272, p. 62721C (2006).

13. L. A. Poyneer, "Advanced techniques for Fourier transform wave-front reconstruction," in Adaptive Optical System Technologies II, P. L. Wizinowich and D. Bonaccini, eds., Proc. SPIE 4839, pp. 1023-1033 (2002). 